\title{
Fabrication of Nano-Structures on NiFe Film by Anodization with Atomic Force Microscope
}

\author{
T. Okada, H. Uchida*, and M. Inoue \\ Department of Electrical and Electronic Engineering, Toyohashi University of Technology, 1-1 Tempaku, Toyohashi 441-8580, Japan
}

(Received 11 September 2006)

\begin{abstract}
We studied local anodization on permalloy $\left(\mathrm{Ni}_{80} \mathrm{Fe}_{20}\right)$ thin film with an atomic force microscope (AFM), which was performed by applying a voltage between the permalloy sample and conductive AFM tip. Comparing with anodization on $\mathrm{Si}(100)$ substrate, nano-structures on the permalloy thin film was fabricated with low processability. In order to improve the processability on the permalloy thin film, we used dot-fabrication method, that is, a conductive AFM probe was kept at a position on the film during the anodization process.
\end{abstract}

Key words : anodization, AFM, probe, permalloy, Si

\section{Introduction}

Scanning probe microscope (SPM), such as a scanning tunneling microscope (STM) and an atomic force microscope (AFM), enables us not only to observe sample surfaces but also to fabricate nano-structures. After the pioneering work on local anodization by Dagata et al. [1], the AFM anodization technique was applied to many different kinds of materials like Si [2], GaAs [3], Al [4] and $\mathrm{Ti}$ [5]. The mechanism of AFM anodization is thought to be local electrochemical reaction on a sample below an AFM tip using water [6]. Since one can fabricate an insulating structure on sample surfaces with a size of $\sim 10 \mathrm{~nm}$ in height and $\sim 100 \mathrm{~nm}$ in width, the technique has attracted much attention as a nano-lithography. In fact, fabrication of electronic device was attempted by using this method [7].

In this paper we reported the results on local anodization of permalloy $\left(\mathrm{Ni}_{80} \mathrm{Fe}_{20}\right)$ thin film. In order to understand mechanism of AFM anodization on permalloy film, we compared results on $\mathrm{Si}(100)$ substrate. We examined the fabrication method to increase processability on the permalloy film.

\section{Experimental}

Fig. 1 shows a schematic of local anodization by AFM.

\footnotetext{
*Corresponding author: Tel: +81-532-44-6731,

Fax: +81-532-44-6757, e-mail: uchida@eee.tut.ac.jp
}

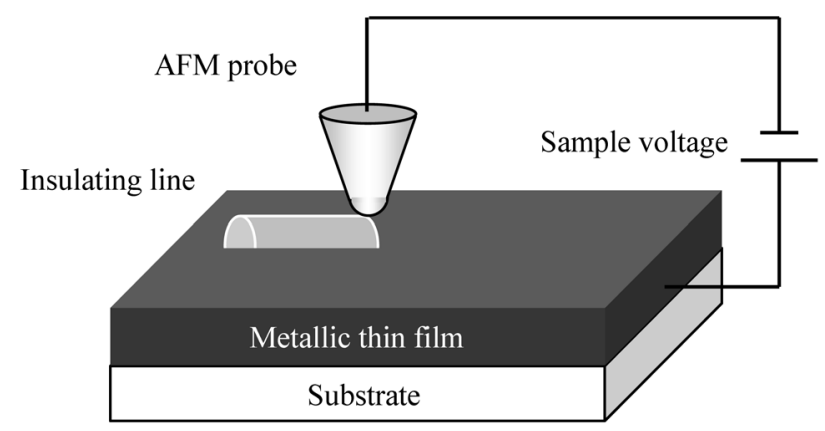

Fig. 1. Schematic of AFM anodization.

Applying a positive voltage to a sample, insulating nanostructure can be formed below a conductive AFM tip. In the experiments, the commercial AFM (JEOL, JSPM5200) and conductive $\mathrm{Si}$ cantilever covered with $\mathrm{Pt} / \mathrm{Ti}$ (MikroMasch) were used. Permalloy $\left(\mathrm{Ni}_{80} \mathrm{Fe}_{20}\right)$ thin films were prepared on n-type $\mathrm{Si}(100)$ substrates by using the RF magnetron sputtering system (Shimadzu, HSR-551). Observation and anodization on the film surfaces were performed by using the tapping (AC) mode of AFM in air with relative humidity of 40 to $50 \%$.

\section{Results and Discussion}

\subsection{AFM anodization on $\mathrm{Si}(100)$ substrate}

On a $\mathrm{Si}(100)$ substrate nano-structures were fabricated with high processability by the AFM anodization. A nano-structure is able to be formed by the contact mode of AFM, however, we used the tapping mode because it 


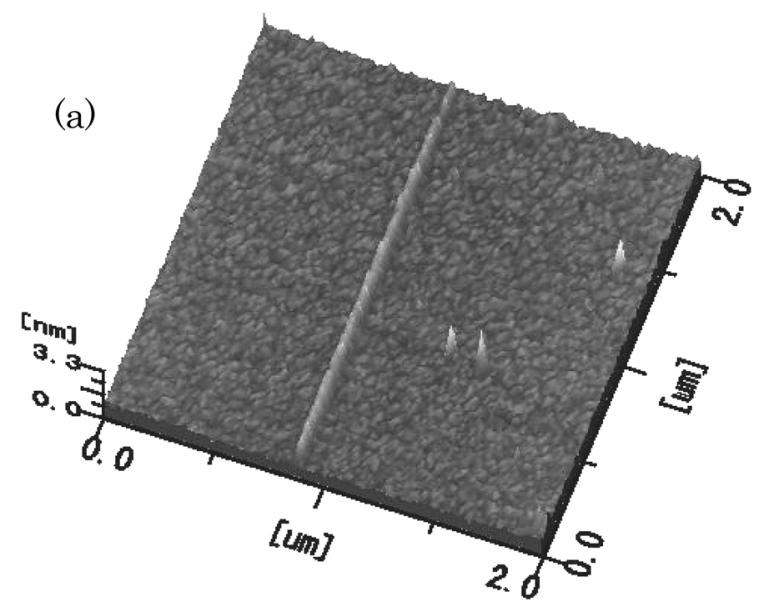

(b)
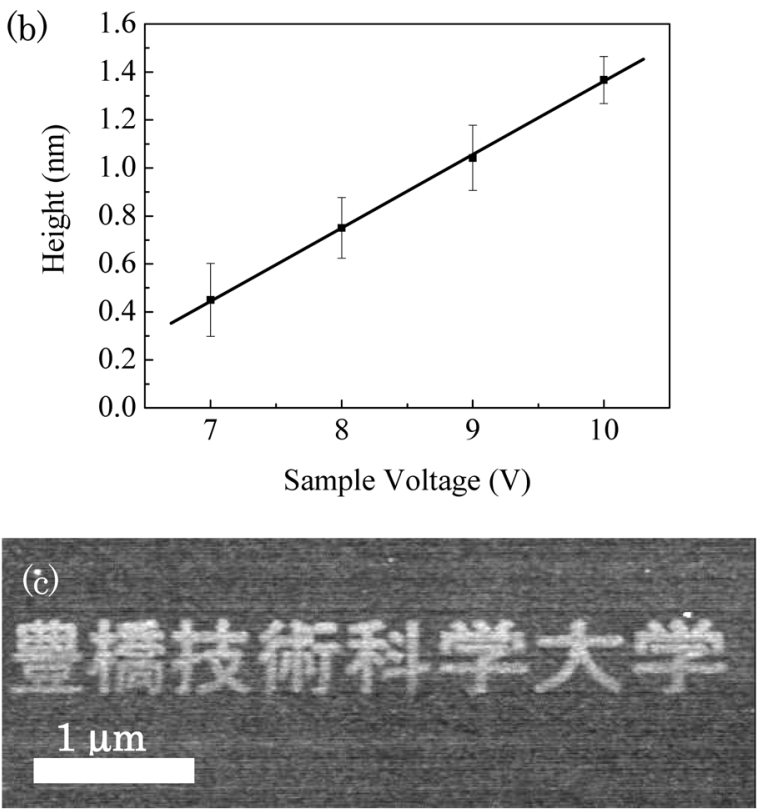

Fig. 2. AFM image of fabricated nano-structures on $\mathrm{Si}(100)$ substrates by AFM anodization. (a) Line structure, (b) heights of fabricated lines as a function of sample voltage and (c) fabricated nano-character.

gives less damage to the AFM tip. Fig. 2(a) shows a line structure on the $\mathrm{Si}(100)$ substrate fabricated by AFM anodization. Height of the line was almost constant, and the continuous structure was fabricated. AFM tip speed was $3 \mu \mathrm{m} / \mathrm{sec}$. Fig. 2(b) shows height of fabricated lines as a function of sample voltage, which an AFM tip was moved with applying voltage during AFM anodization. The height of the line-structures increased linearly by changing sample voltage from 7 to $10 \mathrm{~V}$. That is, the fabricated structure by AFM anodization was controllable by the sample voltage. By using this technique, we tried to make complex structures on the $\mathrm{Si}(100)$ substrate. Fig. 2(c) shows an AFM image of nano-scale characters, which is Japanese name of our university "Toyohashi University

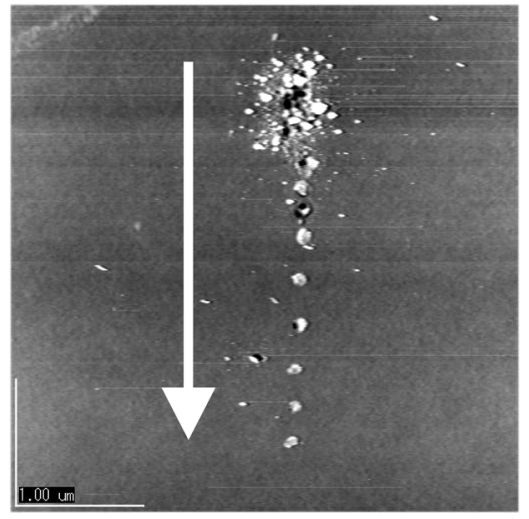

Fig. 3. Attempt of fabrication of line structure on permalloy thin film by AFM anodization.

of Technology". The characters were fabricated by raster tip scan with a voltage of $10 \mathrm{~V}$ and a tip speed of $3 \mu \mathrm{m} /$ sec using previously inputted coordinate data of the characters. The size of each character was $2 \mathrm{~nm}$ in height and $400 \mathrm{~nm}$ in width.

As described above, nano-structures on the $\mathrm{Si}(100)$ substrate were fabricated with high processability; therefore, electrons flowing from conductive AFM tip through a thin native oxide layer of the $\mathrm{Si}$ substrate could be constant during anodization. Flat surface of a substrate can be also required for flowing constant anodization current. In case of the $\mathrm{Si}(100)$ surface, average roughness Ra was $0.12 \mathrm{~nm}$ in the AFM measurement, which is small enough for fabrication of consecutive line by AFM anodization.

\subsection{AFM anodization on permalloy thin film}

Fig. 3 shows an attempt of fabrication of line structure on the $\mathrm{Ni}_{80} \mathrm{Fe}_{20}$ permalloy thin film with a sample voltage of $5 \mathrm{~V}$ and a tip speed of $1 \mu \mathrm{m} / \mathrm{sec}$. A thickness of the permalloy film on the $\mathrm{Si}(100)$ substrate was $30 \mathrm{~nm}$. As a result of this attempt of anodization, irregular structure was formed instead of one line as shown in Fig. 2(a). In experiments for anodization on several kinds of materials we found that processability of anodization largely differs by materials. Namely, the uniform structures were fabricated on the Si substrate, however, on the permalloy, $\mathrm{Fe}$, $\mathrm{Ni}$ and $\mathrm{Co}$ thin films the irregular structures and protrusions were often formed.

In order to improve processability for anodization on the permalloy film, we used dot-fabrication method, which the conductive AFM tip remains stationary during anodization process. In this method the voltage with long duration time at each position was applied. Fig. 4(a) shows an AFM image of two rows of dots with distances of $40 \mathrm{~nm}$ (right side) and $15 \mathrm{~nm}$ (left side) with the 

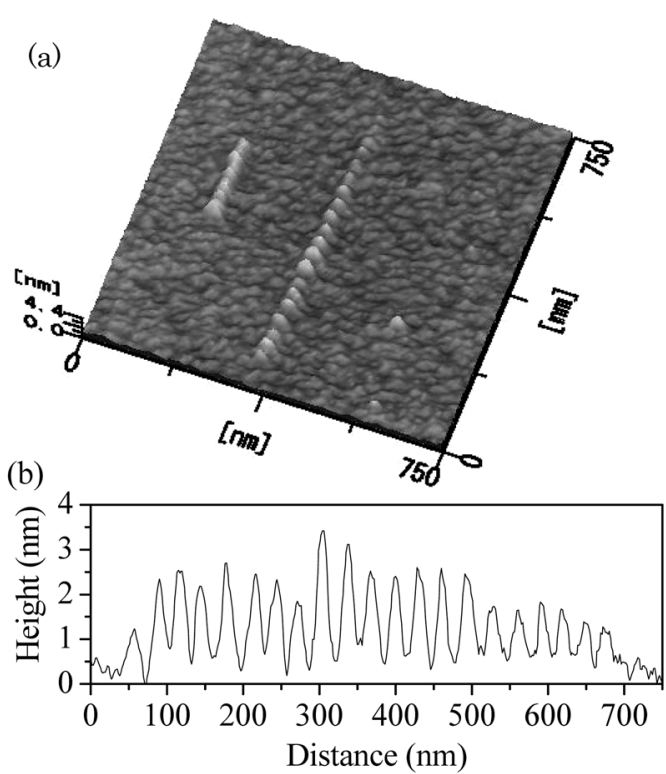

(c)
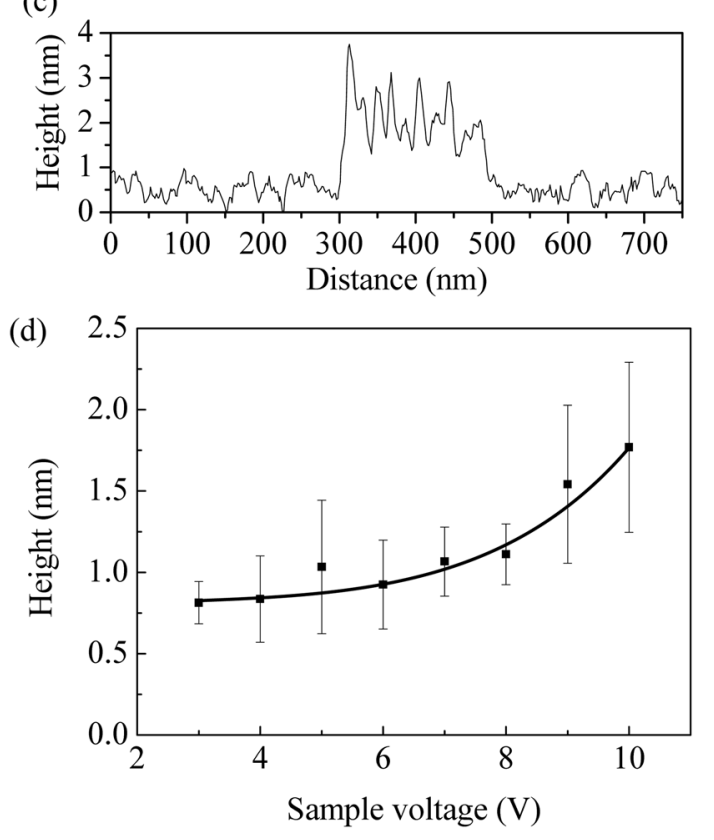

Fig. 4. Fabrication of dot structures by AFM anodization on permalloy thin film. (a) AFM image of dot-structures with distances of $40 \mathrm{~nm}$ (right side) and $15 \mathrm{~nm}$ (left side), (b) a crosssection of dots with distance of $40 \mathrm{~nm}$, (c) a cross-section of dots with distance of $15 \mathrm{~nm}$, and (d) Heights of fabricated dots as a function of sample voltage. The permalloy film after 24 hours from fabrication was used.

duration of $100 \mathrm{~ms}$. By using the dot-fabrication method, processability for anodization on the permalloy film increased in comparison with that of line-fabrication method. Fig. 4(b) shows a cross-section of the row of dots with periodicity of $40 \mathrm{~nm}$ on the permalloy film. Dots were created at all positions where voltages were applied. Although the processability was improved, heights of fabricated dots were not exactly equal. When we applied a voltage in short distance, the structure of connected dots was obtained as seen in Fig. 4(c). Fabrication of dots with shorter distance can produce one line ultimately on the permalloy thin film. Fig. 4(d) shows the heights of fabricated dots as a function of a sample voltage. The thickness of the permalloy film was $30 \mathrm{~nm}$ and average roughness Ra was $0.17 \mathrm{~nm}$. In case of the permalloy film the heights of the dots were not proportional to the sample bias voltage, moreover, standard deviations of heights on the permalloy film were larger than one on the $\mathrm{Si}(100)$ substrate.

Processability of AFM anodization would be influenced by conditions of surface oxide layer such as thickness, stiffness, roughness and so on. During AFM anodization, current flows from a substrate to AFM tip through an oxide layer by the tunneling effect. In our experiments, the uniform structure was fabricated on the $\mathrm{Si}(100)$ substrate (Fig. 2(a)), however, the fabricated structures on the permalloy film was irregular (Fig. 3 and 4). So, nonuniformity of oxide layer on the permalloy film may cause low processability for AFM anodization; it might induce to change the anodization current and the resultant irregular structure on the surface. Otherwise, tip contact during AFM anodization may destroy the oxide layer of permalloy film. Then, flowing large anodization current at a position with the destroyed oxide layer may induce large protrusion on the surface. On the other hand, surface roughness can not be the reason for different processability because both the $\mathrm{Si}(100)$ substrate $(\mathrm{Ra}=0.12 \mathrm{~nm})$ and the permalloy film $(\mathrm{Ra}=0.17 \mathrm{~nm})$ was small enough.

\section{Conclusions}

We investigated AFM anodization on the permalloy film. Fabricated structures on the permalloy film were not uniform in comparison with that on the $\mathrm{Si}(100)$ surface. In order to increase the processability on the permalloy film, we attempted to use dot-fabrication method. However, heights of dots were still inhomogeneous. To improve the processability for fabrication of nano-scale structure on the permalloy thin film, the role of oxide layer of the surface for AFM anodization must be investigated for fabrication of nano-scale magnetic devices.

\section{Acknowledgements}

This work was supported in part by Research Foundation for the Electrotechnology of Chubu, and also Giantin-Aid (C) (No. 17560280) from the Ministry of Education, Culture, Sport and Technology of Japan. 


\section{References}

[1] J. A. Dagata, J. Schneir, H. H. Harary, C. J. Evans, M. T. Postek, and J. Bennett, Appl. Phys. Lett. 56, 2001 (1990).

[2] E. S. Snow and P. M. Campbell, Appl. Phys. Lett. 64, 1932 (1994).

[3] R. Ohashi, T. Ohtsuka, N. Ohta, A. Yamada and M. Konagai, Thin Solid Films 464-465, 237 (2004).
[4] Z. J. Davis, G. Abadal, O. Hansen, X. Borise, N. Barniol, F. Perez-Murano and A. Boisen, Ultramicroscopy 97, 467 (2003).

[5] R. Held, T. Heinzel, P. Studerus and K. Ensslin, Physica E, 2, 748 (1998).

[6] Xiaodong $\mathrm{Hu}$ and Xiaotang Hu, Ultramicroscopy 105, 57 (2005).

[7] Y. Takemura and J. Shirakashi, Advanced Engineering Materials, No.3, 170 (2005). 